\title{
REMAINDER TERMS IN THE FRACTIONAL SOBOLEV INEQUALITY
}

\author{
SHIBING CHEN, RUPERT L. FRANK, AND TOBIAS WETH
}

\begin{abstract}
We show that the fractional Sobolev inequality for the embedding $\stackrel{\circ}{H^{\frac{s}{2}}}\left(\mathbb{R}^{N}\right) \hookrightarrow L^{\frac{2 N}{N-s}}\left(\mathbb{R}^{N}\right), s \in(0, N)$ can be sharpened by adding a remainder term proportional to the distance to the set of optimizers. As a corollary, we derive the existence of a remainder term in the weak $L^{\frac{N}{N-s}}$-norm for functions supported in a domain of finite measure. Our results generalize earlier work for the non-fractional case where $s$ is an even integer.
\end{abstract}

\section{INTRODUCTION}

In this note we consider the fractional Sobolev inequality

$$
\|u\|_{s / 2}^{2} \geq \mathcal{S}\left(\int_{\mathbb{R}^{N}}|u|^{q} d x\right)^{\frac{2}{q}} \quad \text { for all } u \in \stackrel{\circ}{H}^{\frac{s}{2}}\left(\mathbb{R}^{N}\right),
$$

where $0<s<N, q=\frac{2 N}{N-s}$, and $\stackrel{\circ}{H}^{\frac{s}{2}}\left(\mathbb{R}^{N}\right)$ is the space of all tempered distributions $u$ such that

$$
\hat{u} \in L_{l o c}^{1}\left(\mathbb{R}^{N}\right) \quad \text { and } \quad\|u\|_{s / 2}^{2}:=\int_{\mathbb{R}^{N}}|\xi|^{s}|\hat{u}|^{2} d x<\infty .
$$

Here, as usual, $\hat{u}$ denotes the (distributional) Fourier transform of $u$. The best Sobolev constant

$$
\mathcal{S}=\mathcal{S}(N, s)=2^{s} \pi^{\frac{s}{2}} \frac{\Gamma\left(\frac{N+s}{2}\right)}{\Gamma\left(\frac{N-s}{2}\right)}\left(\frac{\Gamma\left(\frac{N}{2}\right)}{\Gamma(N)}\right)^{s / N}
$$

i.e., the largest possible constant in (1), has been computed first in the special case $s=2, N=3$ by Rosen [24] and then independently by Aubin [1] and Talenti 25] for $s=2$ and all dimensions $N$. For general $s \in(0, N)$, the best constant has been given by Lieb [18 for an equivalent reformulation of inequality (1), the (diagonal) Hardy-Littlewood-Sobolev inequality. In order to discuss this equivalence in some more detail, we note that

$$
\|u\|_{s / 2}^{2}=\int_{\mathbb{R}^{N}} u(-\Delta)^{s / 2} u d x
$$

for every Schwartz function $u$, where the operator $(-\Delta)^{s / 2}$ is defined by

$$
\left(\widehat{-\Delta)^{s / 2}} u(\xi)=|\xi|^{s} \widehat{u}(\xi) \quad \text { for a.e. } \xi \in \mathbb{R}^{N} .\right.
$$

Moreover, $\stackrel{\circ}{H}^{\frac{s}{2}}\left(\mathbb{R}^{N}\right)$ is also given as the completion of smooth functions with compact support under the norm $\|\cdot\|_{s / 2}$. The (diagonal) Hardy-Littlewood-Sobolev

Date: May 28, 2012. 
inequality states that

$$
\left|\int_{\mathbb{R}^{N}} \int_{\mathbb{R}^{N}} \frac{f(x) g(y)}{|x-y|^{\lambda}} d x d y\right| \leq \pi^{\lambda / 2} \frac{\Gamma\left(\frac{N-\lambda}{2}\right)}{\Gamma\left(N-\frac{\lambda}{2}\right)}\left(\frac{\Gamma(N)}{\Gamma(N / 2)}\right)^{1-\frac{\lambda}{N}}|f|_{p}|g|_{p}
$$

for all $f, g \in L^{p}\left(\mathbb{R}^{N}\right)$, where $0<\lambda<N$ and $p=\frac{2 N}{2 N-\lambda}$. Here and in the following, we let $|\cdot|_{r}$ denote the usual $L^{r}$-norm for $1 \leq r \leq \infty$. The equivalence of (1) and (41) follows - by a duality argument - from the fact that for every $f \in L^{\frac{q}{q-1}}\left(\mathbb{R}^{N}\right)$ there exists a unique solution $(-\Delta)^{-s / 2} f \in \stackrel{\circ}{H}^{\frac{s}{2}}\left(\mathbb{R}^{N}\right)$ of the equation $(-\Delta)^{s / 2} u=f$ given by convolution with the Riesz potential, i.e., by

$$
\left[(-\Delta)^{-s / 2} f\right](x)=2^{-s} \pi^{-\frac{N}{2}} \frac{\Gamma\left(\frac{N-s}{2}\right)}{\Gamma(s / 2)} \int_{\mathbb{R}^{N}} \frac{1}{|x-y|^{N-s}} f(y) d y \quad \text { for a.e. } x \in \mathbb{R}^{N} .
$$

In [18, Lieb identified the extremal functions for (4), and his results imply that equality holds in (1) for nontrivial $u$ if and only if $u$ is contained in an $N+2$ dimensional submanifold $\mathcal{M}$ of $\stackrel{\circ}{H}^{\frac{s}{2}}\left(\mathbb{R}^{N}\right)$ given as the set of functions which, up to translation, dilation and multiplication by a nonzero constant, coincide with

$$
U \in \stackrel{\circ}{H}^{\frac{s}{2}}\left(\mathbb{R}^{N}\right), \quad U(x)=\left(1+|x|^{2}\right)^{-\frac{N-s}{2}} .
$$

For the special case $s=2$, i.e., the first order Sobolev inequality, Brezis and Lieb [6] asked the question whether a remainder term - proportional to the quadratic distance of the function $u$ to the manifold $\mathcal{M}$ - can be added to the right hand side of (11). This question was answered affirmatively in the case $s=2$ by Bianchi and Egnell [5], and their result was extended later to the case $s=4 \mathrm{in}$ [22] and to the case of an arbitrary even positive integer $s<N$ in [3]. The main purpose of the present note is to obtain a corresponding remainder term inequality for all (real) values $s \in(0, N)$. Our main result is the following.

Theorem 1. Let

$$
\mathcal{M}:=\left\{c U\left(\frac{\cdot-x_{0}}{\varepsilon}\right): c \in \mathbb{R} \backslash\{0\}, x_{0} \in \mathbb{R}^{N}, \varepsilon>0\right\} \subset \stackrel{\circ}{H}^{\frac{s}{2}}\left(\mathbb{R}^{N}\right),
$$

where $U$ is defined in (6). Then there exists a positive constant $\alpha$ depending only on the dimension $N$ and $s \in(0, N)$ such that

$$
d^{2}(u, \mathcal{M}) \geq \int_{\mathbb{R}^{N}} u(-\Delta)^{s / 2}(u) d x-\mathcal{S}\left(\int_{\mathbb{R}^{N}}|u|^{q} d x\right)^{\frac{2}{q}} \geq \alpha d^{2}(u, \mathcal{M})
$$

for all $u \in \stackrel{\circ}{H}^{\frac{s}{2}}\left(\mathbb{R}^{N}\right)$, where $d(u, \mathcal{M})=\min \left\{\|u-\varphi\|_{s / 2}: \varphi \in \mathcal{M}\right\}$.

We briefly explain the strategy to prove this remainder term inequality which goes back to Bianchi and Egnell [5] in the case $s=2$. First, the inequality is proved in a small neighborhood of the optimizer $U \in \mathcal{M}$ defined in (6). Considering a second order Taylor expansion of the difference functional

$$
u \mapsto \Phi(u):=\|u\|_{s / 2}^{2}-\mathcal{S}\left(\int_{\mathbb{R}^{N}}|u|^{q} d x\right)^{\frac{2}{q}},
$$

at $U$, it is not dificult to see that (8) holds in a neighborhood of $U$ with some $\alpha>0$ if and only if the second derivative $\Phi^{\prime \prime}(U)$ is positive definite on the $(N-$ 
2)-codimensional normal space to the manifold $\mathcal{M}$ at $U$. This normal non-degeneracy property is the crucial step in the argument. Once inequality (8) is established in a neighborhood of $U$, it extends to a neighborhood of the whole manifold $\mathcal{M}$ by as a consequence of the conformal invariance of all terms in (7). We will recall this conformal invariance in detail in Section 2 below. Finally, to obtain the global version of (8), a concentration compactness type argument is applied to show that sequences $\left(u_{n}\right)_{n}$ in $\stackrel{\circ}{H}^{\frac{s}{2}}\left(\mathbb{R}^{N}\right)$ with $\Phi\left(u_{n}\right) \rightarrow 0$ as $n \rightarrow \infty$ satisfy $d\left(u_{n}, \mathcal{M}\right) \rightarrow 0$ as $n \rightarrow \infty$.

The general idea described here had already been used in [3,5, 22, but the proofs of the normal non-degeneracy property in these papers strongly rely on the assumption that $s$ is an even positve integer and therefore the eigenvalue problem for $\Phi^{\prime \prime}(U)$ can be written as a differential equation. In particular, ODE arguments are used to study the radial part of the corresponding eigenvalue problem. This method does not apply for general $s \in(0, N)$. On the other hand, one may observe that the eigenvalue problem has a much simpler form once inequality (8) is pulled back on the unit sphere $\mathbb{S}^{N} \subset \mathbb{R}^{N+1}$ via stereographic projection. The equivalent version of Theorem 1 on $\mathbb{S}^{N}$ is given in Theorem 3 below. The idea of studying (1) in its equivalent form on $\mathbb{S}^{N}$ also goes back to Lieb's paper [18] where the (equivalent) Hardy-Littlewood-Sobolev inequality was considered. Afterwards it has been applied in many related problems dealing with Sobolev type inequalities and corresponding Euler-Lagrange equations, see e.g. [2, 4, 9, 23] and the references therein. To our knowledge, its usefulness to identify remainder terms has not been noted so far.

About twenty years after the seminal work of Bianchi and Egnell [5], the topic of remainder terms in first order Sobolev inequalities (and isoperimetric inequalities) has again attracted a lot of attention in the last years. The recent works use techniques from symmetrization (see, e.g., 8, 13), optimal transportation (see, e.g., [12]), and fast diffusion (see, e.g., [10,11, 17]); see also [7] for a recent application of remainder terms. However, while these new methods lead to explicit constants and allow to treat non-Hilbertian Sobolev norms, the estimates for the remainder terms are typically weaker than in the result of Bianchi and Egnell. It is not clear to us to which extent the symmetrization and the optimal transportation approach can be extended to give remainder terms in the higher order case or in the case of arbitrary real powers of the Laplacian (see [17] for a fast diffusion approach in the fractional case). We therefore think it is remarkable that the original strategy of Bianchi-Egnell can be generalized to the full family of conformally invariant Hilbertian Sobolev inequalities.

As a corollary of Theorem [1, we also derive a remainder term inequality for the function space $\stackrel{\circ}{H}^{\frac{s}{2}}(\Omega)$ which - for a subdomain $\Omega \subset \mathbb{R}^{N}$ - is defined as the completion of $\mathcal{C}_{0}^{\infty}(\Omega)$ with respect to the norm $\|\cdot\|_{s / 2}$. In the case where $\Omega$ has a continuous boundary, we have

$$
\stackrel{\circ}{H}^{\frac{s}{2}}(\Omega)=\left\{u \in H^{s}(\Omega): \tilde{u} \in \stackrel{\circ}{H}^{\frac{s}{2}}\left(\mathbb{R}^{N}\right)\right\},
$$


where $\tilde{u}$ denotes the trivial extension of a function $u \in H^{s}(\Omega)$ on $\mathbb{R}^{N}$. We also recall that, for $1<r<\infty$, the weak $L^{r}$-norm of a measurable function $u$ on $\Omega$ is given by

$$
|u|_{w, r, \Omega}=\sup _{\substack{A \subset \Omega \\|A|>0}}|A|^{\frac{1}{r}-1} \int_{A}|u| d x
$$

see e.g. [16.

Theorem 2. Let, as before, $q=\frac{2 N}{N-s}$. Then there exists a constant $C>0$ depending only on $N$ and $s \in(0, N)$ such that for every domain $\Omega \subset \mathbb{R}^{N}$ with $|\Omega|<\infty$ and every $u \in \stackrel{\circ}{H}^{\frac{s}{2}}(\Omega)$ we have

$$
\|u\|_{s / 2}^{2}-\mathcal{S}\left(\int_{\Omega}|u|^{q} d x\right)^{\frac{2}{q}} \geq C|\Omega|^{-\frac{2}{q}}|u|_{w, q / 2, \Omega}^{2}
$$

For fixed bounded domains $\Omega \subset \mathbb{R}^{N}$, the existence of a weak $L^{q / 2}$-remainder term is due to Brezis and Lieb [6] in the case $s=2$ and to Gazzola and Grunau [14] in the case of an arbitrary even positive integer $s<N$. Bianchi and Egnell [5] gave an alternative proof in the case $s=2$ using the corresponding special case of inequality (8). We will follow similar ideas in our proof of Theorem 2. using Theorem 1 in full generality. We note that some additional care is needed to get a remainder term which only depends on $|\Omega|$ and not on $\Omega$ itself.

The paper is organized as follows. In Section 2 we recall the conformal invariance of the problem, and we discuss the framework for an equivalent version of Theorem 1 on the sphere $\mathbb{S}^{N} \subset \mathbb{R}^{N+1}$, see Theorem 3 In Section 3 we prove this Theorem, thus completing the proof of Theorem 1 . In Section 4 we give the proof of Theorem 2

We conclude by pointing out the open problem to find an explicit constant $\alpha>0$ in (8) via a constructive proof of Theorem 1] For a local version of Theorem 1 where the right hand side of (8) is replaced by $\alpha d^{2}(u, \mathcal{M})+o\left(d^{2}(u, \mathcal{M})\right)$ and only $u \in \stackrel{\circ}{H}^{\frac{s}{2}}\left(\mathbb{R}^{N}\right)$ with $d(u, \mathcal{M})<\|u\|_{s / 2}$ is considered, the best constant is $\alpha=\frac{2 s}{N+s+2}$. This follows from Proposition 2 below.

\section{Preliminaries}

In the following, we will denote the scalar product in $\stackrel{\circ}{H}^{\frac{s}{2}}\left(\mathbb{R}^{N}\right)$ by

$$
\langle u, v\rangle_{s / 2}=\int_{\mathbb{R}^{N}}|\xi|^{s} \hat{u}(\xi) \overline{\hat{v}(\xi)} d \xi,
$$

so that $\|u\|_{s / 2}^{2}=\langle u, u\rangle_{s / 2}$ for $u \in \stackrel{\circ}{H}^{\frac{s}{2}}\left(\mathbb{R}^{N}\right)$. In the remainder of this section, $0<s<N$ is fixed and we abbreviate $q=2 n /(N-s)$. We recall that the group of conformal transformations on $\mathbb{R}^{N}$ is generated by translations, rotations, dilations and the inversion $x \mapsto \frac{x}{|x|^{2}}$. If $h$ is one of these transformations with Jacobian determinant $J_{h}$, then for any functions $u, v \in \stackrel{\circ}{H}^{\frac{s}{2}}\left(\mathbb{R}^{N}\right)$ we have $J_{h}^{\frac{1}{q}} u \circ h, J_{h}^{\frac{1}{q}} v \circ h \in$ $\stackrel{\circ}{H}^{\frac{s}{2}}\left(\mathbb{R}^{N}\right)$ and

$$
\left\langle J_{h}^{\frac{1}{q}} u \circ h, J_{h}^{\frac{1}{q}} v \circ h\right\rangle_{s / 2}=\langle u, v\rangle_{s / 2} .
$$


This property is a consequence of the conformal covariance of the operator $(-\Delta)^{s / 2}$, i.e. of the equality

$$
(-\Delta)^{s / 2}\left(J_{h}^{\frac{1}{q}} u \circ h\right)=J_{h}^{\frac{N+s}{2 N}}\left[(-\Delta)^{s / 2} u\right] \circ h
$$

for all conformal transformations $h$ on $\mathbb{R}^{N}$ and all Schwartz functions $u$. As stated in [23, Proposition 2.1], (11) is most easily derived by considering the inverse operator $(-\Delta)^{-s / 2}$ given in (5). Indeed, the identity

$$
(-\Delta)^{-s / 2}\left(J_{h}^{\frac{N+s}{2 N}} u \circ h\right)=J_{h}^{\frac{1}{q}}\left[(-\Delta)^{-s / 2} u\right] \circ h
$$

is equivalent to (11), and it can be verified case by case for dilations, rotations, translations and the inversion. In the latter form related to the Riesz potential, the conformal covariance had already been used by Lieb in 18 .

Note that, if $h$ is a conformal transformation on $\mathbb{R}^{n}$, it follows from (10) that the map $u \mapsto J_{h}^{\frac{1}{q}} u \circ h$ preserves distances with respect to the norm $\|\cdot\|_{s / 2}$, i.e. we have

$$
\left\|J_{h}^{\frac{1}{q}} u \circ h-J_{h}^{\frac{1}{q}} v \circ h\right\|_{s / 2}=\|u-v\|_{s / 2} \quad \text { for all } u, v \in \stackrel{\circ}{H}^{\frac{s}{2}}\left(\mathbb{R}^{N}\right) .
$$

Since the set $\mathcal{M}$ is also invariant under the transformations $u \mapsto J_{h}^{\frac{1}{q}} u \circ h$, we conclude that $d\left(J_{h}^{\frac{1}{q}} u \circ h, \mathcal{M}\right)=d(u, \mathcal{M})$ for all $u \in \stackrel{\circ}{H}^{\frac{s}{2}}\left(\mathbb{R}^{N}\right)$. We also note that

$$
\left|J_{h}^{\frac{1}{q}} u \circ h\right|_{q}=|u|_{q} \quad \text { for any } u \in L^{q}\left(\mathbb{R}^{N}\right)
$$

and any conformal transformation $h$ on $\mathbb{R}^{N}$, which follows by an easy computation. In the following, we consider the inverse stereographic projection

$$
\pi: \mathbb{R}^{N} \rightarrow \mathbb{S}^{N} \subset \mathbb{R}^{N+1}, \quad \pi(x)=\left(\frac{2 x}{1+|x|^{2}}, \frac{1-|x|^{2}}{1+|x|^{2}}\right) .
$$

We recall that $\pi$ is a conformal diffeomorphism. More precisely, if $g_{\mathbb{R}^{N}}$ denotes the flat euclidian metric on $\mathbb{R}^{N}$ and $g_{\mathbb{S}^{N}}$ denotes the metric induced by the embedding $\mathbb{S}^{N} \subset \mathbb{R}^{N+1}$, then the pullback of $g_{\mathbb{S}^{N}}$ to $\mathbb{R}^{N}$ satisfies

$$
\pi^{*} g_{\mathbb{S}^{N}}=\frac{4}{\left(1+|\cdot|^{2}\right)^{2}} g_{\mathbb{R}^{N}}
$$

Moreover, the corresponding volume element is given by

$$
J_{\pi}(x) d x=\left(\frac{2}{1+|x|^{2}}\right)^{N} d x,
$$

For a function $v: \mathbb{S}^{N} \rightarrow \mathbb{R}$, we may now define

$$
\mathcal{P} v: \mathbb{R}^{N} \rightarrow \mathbb{R}, \quad[\mathcal{P} v](x)=J_{\pi}(x)^{\frac{1}{q}} v(\pi(x))=\left(\frac{2}{1+|x|^{2}}\right)^{\frac{N-s}{2}} v(\pi(x)) .
$$

From (16), it is easy to see that $\mathcal{P}$ defines an isometric isomorphism between $L^{q}\left(\mathbb{S}^{N}\right)$ and $L^{q}\left(\mathbb{R}^{N}\right)$. We also note that

$$
\mathcal{P} 1=2^{(N-s) / 2} U,
$$

where 1 stands for unit function on $\mathbb{S}^{N}$ and $U$ is defined in (6). Moreover, $H^{\frac{s}{2}}\left(\mathbb{S}^{N}\right)$ is the completion of the space of smooth functions on $\mathbb{S}^{N}$ under the norm $\|\cdot\|_{*}$ 
induced by scalar product

$$
(u, v) \mapsto\langle u, v\rangle_{*}=\langle\mathcal{P} u, \mathcal{P} v\rangle_{s / 2}
$$

We will always consider $H^{\frac{s}{2}}\left(\mathbb{S}^{N}\right)$ with the norm $\|\cdot\|_{*}$ induced by this scalar product (for matters of convenience, we suppress the dependence on $s$ at this point). Hence, by construction,

$\mathcal{P}$ is also an isometric isomorphism $\left(H^{\frac{s}{2}}\left(\mathbb{S}^{N}\right),\|\cdot\|_{*}\right) \rightarrow\left(\stackrel{\circ}{H^{\frac{s}{2}}}\left(\mathbb{R}^{N}\right),\|\cdot\|_{s / 2}\right)$.

Next we note that $\langle\cdot, \cdot\rangle_{*}$ is the quadratic form of a unique positive self adjoint operator in $L^{2}\left(\mathbb{S}^{N}\right)$ which is commonly denoted by $A_{s}$ in the literature. This operator is formally given by

$$
\left[A_{s} w\right] \circ \pi=J_{\pi}^{-\frac{N+s}{2 N}}(-\Delta)^{s / 2}(\mathcal{P} w) .
$$

A key ingredient of the proof of Theorem 1 is the following representation of $A_{s}$ as a function of the Laplace-Beltrami Operator $\Delta_{\mathbb{S}^{N}}$ on $\mathbb{S}^{N}$ :

$$
A_{s}=\frac{\Gamma\left(B+\frac{1+s}{2}\right)}{\Gamma\left(B+\frac{1-s}{2}\right)} \quad \text { with } B=\sqrt{-\Delta_{\mathbb{S}^{N}}+\left(\frac{N-1}{2}\right)^{2}} .
$$

This formula is most easily derived by considering the inverse of $A_{s}$ and using the Funk-Hecke formula, see 4] and also [23. It also shows that the domain of $A_{s}$ coincides with $H^{s}\left(\mathbb{S}^{N}\right)$. The following statement is a mere reformulation of (18).

Proposition 1. The operator $A_{s}$ is self adjoint and has compact resolvent. Its spectrum is given as the sequence of eigenvalues

$$
\lambda_{k}(s)=\frac{\Gamma\left(\frac{N+s}{2}+k\right)}{\Gamma\left(\frac{N-s}{2}+k\right)}, \quad k \in \mathbb{N}_{0},
$$

and the eigenspace corresponding to the eigenvalue $\lambda_{k}(s)$ is spanned by the spherical harmonics $Y_{k, j}, j=1, \ldots,\left(\begin{array}{c}k+N \\ N\end{array}\right)-\left(\begin{array}{c}k+N-2 \\ N\end{array}\right)$, of degree $k$.

Next, we note that, via the isometric isomorphism $\mathcal{P}$, inequality (11) is equivalent to

$$
\|u\|_{*}^{2} \geq \mathcal{S}|u|_{q}^{2} \quad \text { for all } u \in H^{\frac{s}{2}}\left(\mathbb{S}^{N}\right)
$$

with $q=\frac{2 N}{N-s}$. Here, in accordance with the previous notation, we also write $|\cdot|_{r}$ for the $L^{r}$-norm of a function in $L^{r}\left(\mathbb{S}^{N}\right), 1 \leq r \leq \infty$. Equality is attained in (19) for nontrivial $u$ if and only if $u \in \mathcal{M}_{*}$, where

$$
\mathcal{M}_{*}:=\mathcal{P}^{-1}(\mathcal{M})=\left\{v \in H^{\frac{s}{2}}\left(\mathbb{S}^{N}\right): \mathcal{P} v \in \mathcal{M}\right\}
$$

Moreover, the remainder term inequality (8) is equivalent to

$$
d^{2}\left(u, \mathcal{M}_{*}\right) \geq\|u\|_{*}^{2}-\mathcal{S}|u|_{q}^{2} \geq \alpha d^{2}\left(u, \mathcal{M}_{*}\right) \quad \text { for } u \in H^{s / 2}\left(\mathbb{S}^{N}\right)
$$

where $d\left(u, \mathcal{M}_{*}\right)=\min \left\{\|u-\varphi\|_{*}: \varphi \in \mathcal{M}\right\}$. We may therefore reformulate Theorem 1 as follows.

Theorem 3. There exists a positive constant $\alpha$ depending only on the dimension $N$ and $s \in(0, N)$ such that (20) holds. 
We will prove Theorem 3 in Section 3 below, thus completing the proof of Theorem 1. We close this section with some comments on the conformal invariance of the reformulated problem and the geometry of $\mathcal{M}_{*}$. Via stereographic projection, the conformal transformations on $\mathbb{S}^{N}$ are in 1-1-correspondance with the conformal transformations on $\mathbb{R}^{N}$. So, if $\tau$ is an element of the conformal group of $\mathbb{S}^{N}$ with Jacobian determinant $J_{\tau}$, then (14) and (10) imply that

$$
\left\langle J_{\tau}^{\frac{1}{q}} u \circ \tau, J_{\tau}^{\frac{1}{q}} v \circ \tau\right\rangle_{s / 2}=\langle u, v\rangle_{*} \quad \text { and } \quad\left|J_{h}^{\frac{1}{q}} u \circ h\right|_{q}=|u|_{q}
$$

for all $u, v \in H^{\frac{s}{2}}\left(\mathbb{S}^{N}\right)$. From (17), we deduce the representation

$$
\mathcal{M}_{*}=\left\{c J_{\tau}^{\frac{1}{q}} \mid \tau \text { is an element of the conformal group of } S^{N}, c \in \mathbb{R} \backslash\{0\}\right\} .
$$

Since the Jacobian determinant $J_{\tau}$ of a conformal transformation $\tau$ on $\mathbb{S}^{N}$ has the form $J_{\tau}(\xi)=(1-\xi \cdot \theta)^{-n}$ for some $\theta \in B^{n+1}:=\left\{x \in \mathbb{R}^{N+1}:|x|<1\right\}, \mathcal{M}_{*}$ can be viewed as an $N+2$ dimensional smooth manifold embedded in $H^{\frac{s}{2}}\left(\mathbb{S}^{N}\right)$ via the mapping

$$
\mathbb{R} \backslash\{0\} \times B^{N+1} \rightarrow H^{\frac{s}{2}}\left(\mathbb{S}^{N}\right), \quad(c, \theta) \mapsto u_{c, \theta},
$$

where $u_{c, \theta}(\xi)=c(1-\xi \cdot \theta)^{-\frac{N-s}{2}}$ for $\xi \in \mathbb{S}^{N}$. This immediately implies that the tangent space $T_{1} \mathcal{M}_{*}$ at the function $1=u_{1,0}$ is generated by the spherical harmonics $Y_{0}^{0}=1$ and $Y_{1}^{j}, j=1, \ldots, N+1$, given by

$$
Y_{1}^{j}(\xi)=\xi_{j} \quad \text { for } \xi=\left(\xi_{1}, \ldots, \xi_{N+1}\right) \in \mathbb{S}^{N} \subset \mathbb{R}^{N+1} .
$$

Hence $T_{1} \mathcal{M}_{*}$ coincides precisely with the generalized eigenspace of the operator $A_{s}$ corresponding to the eigenvalues $\lambda_{0}(s)$ and $\lambda_{1}(s)$. Combining this fact with the minimax characterization of the eigenvalue $\lambda_{2}(s)$, we readily deduce that

$$
\lambda_{2}(s)=\inf _{v \in T_{1} \mathcal{M}_{*}^{\perp}} \frac{\|v\|^{2}}{|v|_{2}^{2}}
$$

with

$$
T_{1} \mathcal{M}_{*}^{\perp}:=\left\{v \in H^{\frac{s}{2}}\left(\mathbb{S}^{N}\right):\langle v, w\rangle_{*}=0 \text { for all } w \in T_{1} \mathcal{M}_{*}\right\} .
$$

The identity (23) will be of crucial importance for the local verification of (20) close to the manifold $\mathcal{M}_{*}$.

\section{Proof of the Remainder term InEQuality on the SPHERE}

We first prove a local variant of Theorem 3.

Proposition 2. For all $u \in H^{\frac{s}{2}}\left(\mathbb{S}^{N}\right)$ with $d\left(u, \mathcal{M}_{*}\right)<\|u\|_{*}$, we have

$$
d^{2}\left(u, \mathcal{M}_{*}\right) \geq\|u\|_{*}^{2}-\mathcal{S}|u|_{q}^{2} \geq \frac{2 s}{N+s+2} d^{2}\left(u, \mathcal{M}_{*}\right)+o\left(d^{2}\left(u, \mathcal{M}_{*}\right)\right) .
$$

Proof. We consider the functional

$$
\Psi: H^{\frac{s}{2}}\left(\mathbb{S}^{N}\right) \rightarrow \mathbb{R}, \quad \Psi(u)=\|u\|_{*}^{2}-\mathcal{S}|u|_{q}^{2} .
$$

It is easy to see that $\Psi$ is of class $\mathcal{C}^{2}$ on $H^{\frac{s}{2}}\left(\mathbb{S}^{N}\right) \backslash\{0\}$. Moreover,

$$
\Psi^{\prime}(u) v=2\langle u, v\rangle_{*}-2 \mathcal{S}|u|_{q}^{2-q} \int_{\mathbb{S}^{N}}|u|^{q-2} u v d \xi
$$


and

$$
\begin{aligned}
\frac{1}{2} \Psi^{\prime \prime}(u)(v, w)=\langle v, w\rangle_{*} & -\mathcal{S}(2-q)|u|_{q}^{2-2 q} \int_{\mathbb{S}^{N}}|u|^{q-2} u v d \xi \int_{\mathbb{S}^{N}}|u|^{q-2} u w d \xi \\
& -\mathcal{S}(q-1)|u|_{q}^{2-q} \int_{\mathbb{S}^{N}}|u|^{q-2} v w d \xi
\end{aligned}
$$

for $u \in H^{\frac{s}{2}}\left(\mathbb{S}^{N}\right) \backslash\{0\}, v, w \in H^{\frac{s}{2}}\left(\mathbb{S}^{N}\right)$.

Next, let $u \in H^{\frac{s}{2}}\left(\mathbb{S}^{N}\right)$ with $d\left(u, \mathcal{M}_{*}\right)<\|u\|_{*}$. It is easy to see that $d\left(u, \mathcal{M}_{*}\right)$ is achieved by some function $c J_{\tau}^{\frac{1}{q}}$ in $\mathcal{M}_{*}$ with $c \in \mathbb{R} \backslash\{0\}$ and a conformal transformation $\tau$ on $\mathbb{S}^{N}$. Replacing $u$ with $\frac{1}{c} J_{\tau^{-1}}^{\frac{1}{q}} u \circ \tau^{-1}$ and using (21), we may assume that $c=1$ and $\tau=\mathrm{id}$, hence we may write $u=1+v$ with $v \in T_{1} \mathcal{M}_{*}^{\perp}$, the normal space of $\mathcal{M}_{*}$ at 1 defined in (24), and $d\left(u, \mathcal{M}_{*}\right)=\|v\|_{*}$. We note that $\Psi(1)=0$ and $\Psi^{\prime}(1)=0$ (since the function 1 is a global minimizer of $\Psi$ ). Moreover, the condition $v \in T_{1} \mathcal{M}_{*}^{\perp}$ in particular implies - since $1 \in T_{1} \mathcal{M}_{*}$ - that

$$
\langle 1, v\rangle_{*}=0 \quad \text { and } \quad \int_{\mathbb{S}^{N}} v d \xi=0 .
$$

In particular, we find that

$$
\begin{aligned}
\Psi(u) & =\Psi(1+v)=\|1\|_{*}^{2}+\|v\|_{*}^{2}-\mathcal{S}|1+v|_{q}^{2} \leq\|1\|_{*}^{2}+\|v\|_{*}^{2}-\mathcal{S}\left|\mathbb{S}^{N}\right|^{\frac{2-q}{q}}|1+v|_{2}^{2} \\
& =\|1\|_{*}^{2}+\|v\|_{*}^{2}-\mathcal{S}\left|\mathbb{S}^{N}\right|^{\frac{2-q}{q}}\left(\left|\mathbb{S}^{N}\right|+|v|_{2}^{2}\right)=\Psi(1)+\|v\|_{*}^{2}-\mathcal{S}\left|\mathbb{S}^{N}\right|^{\frac{2-q}{q}}|v|_{2}^{2} \\
& \leq\|v\|_{*}^{2}=d^{2}\left(u, \mathcal{M}_{*}\right),
\end{aligned}
$$

and this yields the first inequality in (25). Moreover, from (28) and (29) we infer that

$$
\frac{1}{2} \Psi^{\prime \prime}(1)(v, v)=\|v\|_{*}^{2}-(q-1) \mathcal{S}\left|\mathbb{S}^{N}\right|^{\frac{2-q}{q}} \int_{\mathbb{S}^{N}} v^{2} d \xi
$$

A second order Taylor expansion of $\Psi$ at 1 thus yields

$$
\begin{aligned}
\Psi(u)=\Psi(1+v) & =\frac{1}{2} \Psi^{\prime \prime}(1)(v, v)+o\left(\|v\|_{*}^{2}\right) \\
& =\|v\|_{*}^{2}-(q-1) \mathcal{S}\left|\mathbb{S}^{N}\right|^{\frac{2-q}{q}}|v|_{2}^{2}+o\left(\|v\|_{*}^{2}\right) .
\end{aligned}
$$

Using (21) and the identity $\left|\mathbb{S}^{N}\right|=2 \pi^{\frac{N+1}{2}} \Gamma\left(\frac{N+1}{2}\right)^{-1}$, we find by a short computation (using the duplication formula for the Gamma function) that

$$
(q-1) \mathcal{S}\left|\mathbb{S}^{N}\right|^{\frac{2-q}{q}}=\frac{N+s}{N-s} \mathcal{S}\left|\mathbb{S}^{N}\right|^{-\frac{s}{N}}=\frac{\Gamma\left(\frac{N+s}{2}+1\right)}{\Gamma\left(\frac{N-s}{2}+1\right)}=\lambda_{1}(s) .
$$

Noting moreover that $|v|_{2}^{2} \leq \frac{\|v\|_{*}^{2}}{\lambda_{2}(s)}$ as a consequence of (23), we conclude that

$$
\Psi(u) \geq\|v\|_{*}^{2}\left(1-\frac{\lambda_{1}(s)}{\lambda_{2}(s)}+o(1)\right)=d\left(u, \mathcal{M}_{*}\right)^{2}\left(\frac{2 s}{N+s+2}+o(1)\right)
$$

This shows the second inequality in (25).

The next tool we need is the following property of optimizing sequences for (1).

Lemma 1. Let $\left(u_{m}\right)_{m} \subset \stackrel{\circ}{H^{\frac{s}{2}}}\left(\mathbb{R}^{N}\right) \backslash\{0\}$ be a sequence with $\lim _{m \rightarrow \infty} \frac{\left\|u_{m}\right\|_{*}^{2}}{\left|u_{m}\right|_{q}^{2}}=\mathcal{S}$. Then $\frac{d\left(u_{m}, \mathcal{M}_{*}\right)}{\left\|u_{m}\right\|_{*}} \rightarrow 0$ as $m \rightarrow \infty$. 
Proof. By homogeneity, we may assume that $\left\|u_{m}\right\|_{*}=1$ for all $m \in \mathbb{N}$, and we need to show that $d\left(u_{m}, \mathcal{M}_{*}\right) \rightarrow 0$ as $m \rightarrow \infty$. We let $v_{m}=\mathcal{P} u_{m} \in \stackrel{\circ}{H}^{\frac{s}{2}}\left(\mathbb{R}^{N}\right)$ for $m \in \mathbb{N}$; then $\left\|v_{m}\right\|_{s / 2}=1$ for all $m$, and

$$
\frac{1}{\left|v_{m}\right|_{q}^{2}} \rightarrow \mathcal{S} \quad \text { as } m \rightarrow \infty
$$

By the profile decomposition theorem of Gérard (see [15, Théorème 1.1 and Remarque 1.2]), there exists a subsequence - still denoted by $\left(v_{m}\right)_{m}$ - and

- a sequence $\left(\psi_{j}\right)_{j}$ of functions $\psi_{j} \in \stackrel{\circ}{H^{\frac{s}{2}}}\left(\mathbb{R}^{N}\right)$,

- an increasing sequence of numbers $l_{m} \in \mathbb{N}, m \in \mathbb{N}$,

- a double sequence of values $h_{m}^{j} \in(0, \infty), m, j \in \mathbb{N}$,

- a double sequence of points $x_{m}^{j} \in \mathbb{R}^{N}, m, j \in \mathbb{N}$

such that

$$
\begin{aligned}
& \left|v_{m}-\sum_{j=1}^{l_{m}}\left(h_{m}^{j}\right)^{-\frac{s}{2 q}} \psi_{j}\left(\frac{\cdot-x_{m}^{j}}{h_{m}^{j}}\right)\right|_{q} \rightarrow 0 \quad \text { as } m \rightarrow \infty, \\
& \left|v_{m}\right|_{q}^{q} \rightarrow \sum_{j=1}^{\infty}\left|\psi_{j}\right|_{q}^{q} \quad \text { as } m \rightarrow \infty \quad \text { and } \quad \sum_{j=1}^{\infty}\left\|\psi_{j}\right\|_{s / 2}^{2} \leq 1 .
\end{aligned}
$$

Combining the Sobolev inequality (1) with (32) and using the concavity of the function $t \mapsto t^{2 / q}$, we find that

$$
1 \geq \mathcal{S} \sum_{j=1}^{\infty}\left|\psi_{j}\right|_{q}^{2} \geq \mathcal{S}\left(\sum_{j=1}^{\infty}\left|\psi_{j}\right|_{q}^{q}\right)^{2 / q}=\mathcal{S} \lim _{m \rightarrow \infty}\left|v_{m}\right|_{q}^{2} .
$$

By (30), equality holds in all steps in (33). The strict concavity of the function $t \mapsto t^{2 / q}$ then shows that $\psi_{j} \equiv 0$ for all but one $j \in \mathbb{N}$, say, $j=1$, where $\mathcal{S}\left|\psi_{1}\right|_{q}^{2}=1$ and $\left\|\psi_{1}\right\|_{s / 2}=1$ as a consequence of (32), (33) and the Sobolev inequality (1). Hence $\Psi_{1} \in \mathcal{M}$, and from (31) it now follows that

$$
\left|v_{m}-\left(h_{m}^{1}\right)^{-\frac{s}{2 q}} \psi_{1}\left(\frac{\cdot-x_{m}^{1}}{h_{m}^{1}}\right)\right|_{q} \rightarrow 0 \quad \text { as } m \rightarrow \infty .
$$

Therefore, defining

$$
\tilde{v}_{m} \in \stackrel{\circ}{H}^{\frac{s}{2}}\left(\mathbb{R}^{N}\right), \quad \tilde{v}_{m}(x)=\left(h_{m}^{1}\right)^{\frac{s}{2 q}} v_{m}\left(h_{m}^{1} x+x_{m}^{1}\right) \quad \text { for } m \in \mathbb{N},
$$

we have $\tilde{v}_{m} \rightarrow \psi_{1}$ in $L^{q}\left(\mathbb{R}^{N}\right)$ for $m \rightarrow \infty$, but then also $\tilde{v}_{m} \rightarrow \psi_{1}$ in $\stackrel{\circ}{H}^{\frac{s}{2}}\left(\mathbb{R}^{N}\right)$ strongly since $\left\|\tilde{v}_{m}\right\|_{s / 2}=\left\|v_{m}\right\|_{s / 2}=1=\left\|\psi_{1}\right\|_{s / 2}$ for all $m \in \mathbb{N}$. Consequently, $d\left(\tilde{v}_{m}, \mathcal{M}\right) \rightarrow 0$. By the invariance property (13), we then have $d\left(v_{m}, \mathcal{M}\right) \rightarrow 0$ and therefore also $d\left(u_{m}, \mathcal{M}_{*}\right) \rightarrow 0$ as $m \rightarrow \infty$, since $\mathcal{P}$ is an isometry.

Remark 1. (i) We note that we do not need the full strength of Gérard's profile decomposition theorem. Inductively, Gérard writes $v_{m}$ as an infinite sum of bubbles, see (31) and 15. For our proof it is enough to stop this procedure after the very first step. As soon as one bubble is extracted, the strict concavity of the function $t \mapsto t^{2 / q}$ implies the convergence.

(ii) In the case where $s \in(0, N)$ is an even integer, one could also use a classical concentration compactness result of Lions instead of Gérard's result, see [20, Corollary 1]. 
(iii) For arbitrary $s \in(0, N)$, one could also use the duality between (1) and (4) explained in the introduction and another concentration compactness result of Lions about optimizing sequences for (4), see [21, Theorem 2.1]. To us it seemed more natural to use a technique directly applicable to optimizing sequences for (1).

With the help of Proposition 2 and Lemma 1, we may now complete the

Proof of Theorem [3. Let $u \in H^{\frac{s}{2}}\left(\mathbb{S}^{N}\right)$. Since $0 \in \overline{\mathcal{M}_{*}}$, we have $d\left(u, \mathcal{M}_{*}\right) \leq\|u\|_{*}$. If $d\left(u, \mathcal{M}_{*}\right)<\|u\|_{*}$, then the first inequality in (20) follows from Proposition 2, and it is trivially satisfied if $d\left(u, \mathcal{M}_{*}\right)=\|u\|_{*}$. To prove the second inequality in (20) for some $\alpha>0$, we argue by contradiction. For this we assume that there exists a sequence $\left(u_{m}\right)_{m}$ in $H^{\frac{s}{2}}\left(\mathbb{S}^{N}\right) \backslash \overline{\mathcal{M}_{*}}$ with

$$
\frac{\left\|u_{m}\right\|_{*}^{2}-\mathcal{S}\left|u_{m}\right|_{q}^{2}}{d^{2}\left(u_{m}, \mathcal{M}_{*}\right)} \rightarrow 0 \quad \text { as } m \rightarrow \infty .
$$

By homogeneity we can assume that $\left\|u_{m}\right\|_{*}=1$ for all $m \in \mathbb{N}$, then $d\left(u_{m}, \mathcal{M}_{*}\right) \leq 1$ for all $m \in \mathbb{N}$ and therefore (34) implies that $\lim _{m \rightarrow \infty}\left|u_{m}\right|_{q}^{2}=\frac{1}{\mathcal{S}}$. Hence Lemma 1 gives $d\left(u_{m}, \mathcal{M}\right) \rightarrow 0$ as $m \rightarrow \infty$. But then Proposition 2 shows that (34) must be false. We conclude that there exists $\alpha>0$ such that

$$
\|u\|_{*}^{2}-\mathcal{S}|u|_{q}^{2} \geq \alpha d^{2}\left(u, \mathcal{M}_{*}\right) \quad \text { for all } u \in H^{\frac{s}{2}}\left(\mathbb{S}^{N}\right)
$$

as claimed.

4. The WEAK $L^{q / 2}$ REMAINDER TERM INEQUALity FOR DOMAINS OF FINITE MEASURE

In this section we give the proof of Theorem 2 For this we define

$$
U_{\lambda, y} \in \stackrel{\circ}{H}^{\frac{s}{2}}\left(\mathbb{R}^{N}\right), \quad U_{\lambda, y}(x):=\lambda U\left(\lambda^{\frac{2}{N-s}}(x-y)\right)
$$

for $c \in \mathbb{R} \backslash\{0\}, \lambda>0$ and $y \in \mathbb{R}^{N}$, so that

$$
\mathcal{M}=\left\{c U_{\lambda, y}: c \in \mathbb{R} \backslash\{0\}, \lambda>0, y \in \mathbb{R}^{N}\right\} .
$$

It will be convenient to adjust the notation for the weak $L^{q / 2}$-norm. We fix $q=\frac{2 N}{N-s}$ from now on, and we write

$$
|u|_{w, \Omega}=\sup _{\substack{A \subset \Omega \\|A|>0}}|A|^{-\frac{s}{N}} \int_{A}|u| d x .
$$

for the weak $L^{q / 2}$-norm of a measurable function $u$ defined on a measurable set $\Omega \subset$ $\mathbb{R}^{N}$. We note the following scaling property, which follows by direct computation:

$$
\left|U_{\lambda, y}\right|_{w, \mathbb{R}^{N}}=\left|U_{\lambda, 0}\right|_{w, \mathbb{R}^{N}}=\frac{|U|_{w, \mathbb{R}^{N}}}{\lambda} \quad \text { for } \lambda>0, y \in \mathbb{R}^{N} .
$$

Similarly, for a fixed domain $\Omega \subset \mathbb{R}^{N}, u \in \stackrel{\circ}{H}^{\frac{s}{2}}(\Omega)$ and $\lambda>0$, define

$$
\Omega_{\lambda}:=\lambda^{-2 /(N-s)} \Omega \subset \mathbb{R}^{N} \quad \text { and } \quad u_{\lambda} \in \stackrel{\circ}{H}^{\frac{s}{2}}\left(\Omega_{\lambda}\right), \quad u_{\lambda}(x)=\lambda u\left(\lambda^{\frac{2}{N-s}} x\right) .
$$

Then a direct computation shows

$$
\left|\Omega_{\lambda}\right|=\lambda^{-q}|\Omega|, \quad\left|u_{\lambda}\right|_{w, \Omega_{\lambda}}=\frac{|u|_{w, \Omega}}{\lambda} \quad \text { and } \quad d\left(u_{\lambda}, \mathcal{M}\right)=d(u, \mathcal{M}) .
$$


Theorem 2 will follow immediately from the following Proposition.

Proposition 3. There exists a constant $C_{0}$ depending only on $N$ and $s \in(0, N)$ such that

$$
|u|_{w, \Omega} \leq C_{0}|\Omega|^{\frac{1}{q}} d(u, \mathcal{M})
$$

for all subdomains $\Omega \subset \mathbb{R}^{N}$ with $|\Omega|<\infty$ and all $u \in \stackrel{\circ}{H}^{\frac{s}{2}}(\Omega)$.

Proof. By the scaling properties noted in (36), it suffices to consider a subdomain $\Omega \subset \mathbb{R}^{N}$ with $|\Omega|=1$ in the sequel. In this case we have, by Hölder's inequality and (11),

$$
|u|_{w, \Omega} \leq\|u\|_{L^{q}(\Omega)} \leq\|u\|_{L^{q}\left(\mathbb{R}^{N}\right)} \leq \frac{1}{\sqrt{\mathcal{S}}}\|u\|_{s / 2} \quad \text { for every } u \in \stackrel{\circ}{H^{\frac{s}{2}}}\left(\mathbb{R}^{N}\right) .
$$

In the following, let $\rho \in(0,1)$ be given by

$$
\frac{\rho}{\sqrt{\mathcal{S}}(1-\rho)}=\left(\left|\mathbb{S}^{N-1}\right| \int_{1}^{\infty} \frac{r^{N-1}}{\left(1+r^{2}\right)^{N}} d r\right)^{\frac{1}{q}}
$$

Let $u \in \stackrel{\circ}{H}^{\frac{s}{2}}(\Omega)$. If $\rho\|u\|_{s / 2} \leq d(u, \mathcal{M})$, then

$$
|u|_{w, \Omega} \leq \frac{1}{\rho \sqrt{\mathcal{S}}} d(u, \mathcal{M})
$$

as a consequence of (38). So in the remainder of this proof we assume that

$$
\rho\|u\|_{s / 2}>d(u, \mathcal{M}) .
$$

By homogeneity we may assume that $\|u\|_{s / 2}=1$. Since $\rho<1$, the infimum in the definition of $d(u, \mathcal{M})$ is attained as a consequence of (41), and we have $d(u, \mathcal{M})=\left\|u-c U_{\lambda, y}\right\|_{s / 2}$ for some $c \in \mathbb{R}, \lambda>0$ and $y \in \mathbb{R}^{n}$. Moreover, (41) implies that

$$
|1-c|=\left|\|u\|_{s / 2}-\left\|c U_{\lambda, y}\right\|_{s / 2}\right| \leq d(u, \mathcal{M}) \leq \rho,
$$

that is, $1-\rho \leq c \leq 1+\rho$. We note that

$$
\begin{aligned}
d(u, \mathcal{M})^{2} & =\left\|u-c U_{\lambda, y}\right\|_{s / 2}^{2} \geq \mathcal{S}\left\|u-c U_{\lambda, y}\right\|_{L^{q}\left(\mathbb{R}^{N}\right)}^{2} \\
& \geq \mathcal{S}|c|^{2}\left\|U_{\lambda, y}\right\|_{L^{q}\left(\mathbb{R}^{N} \backslash \Omega\right)}^{2} \geq \mathcal{S}(1-\rho)^{2}\left\|U_{\lambda, y}\right\|_{L^{q}\left(\mathbb{R}^{N} \backslash \Omega\right)}^{2} .
\end{aligned}
$$

Now let $B \subset \mathbb{R}^{N}$ denote the open ball centered at zero with $|B|=1$, and let $r_{0}>0$ denote the radius of $B$. Since the function $U$ in (6) is radial and strictly decreasing in the radial variable, the bathtub principle [19, Theorem 1.14] implies that

$$
\left\|U_{\lambda, y}\right\|_{L^{q}\left(\mathbb{R}^{N} \backslash \Omega\right)}^{2} \geq\left\|U_{\lambda, y}\right\|_{L^{q}\left(\mathbb{R}^{N} \backslash(B+y)\right)}^{2}=\left\|U_{\lambda, 0}\right\|_{L^{q}\left(\mathbb{R}^{N} \backslash B\right)}^{2},
$$

and hence

$$
\left\|U_{\lambda, 0}\right\|_{L^{q}\left(\mathbb{R}^{N} \backslash B\right)}^{q} \leq\left(\frac{d(u, \mathcal{M})}{\sqrt{\mathcal{S}}(1-\rho)}\right)^{q} \leq\left(\frac{\rho}{\sqrt{\mathcal{S}}(1-\rho)}\right)^{q}=\left|\mathbb{S}^{N-1}\right| \int_{1}^{\infty} \frac{r^{N-1}}{\left(1+r^{2}\right)^{N}} d r
$$

by our choice of $\rho$ in (39). On the other hand, we compute

$$
\left\|U_{\lambda, 0}\right\|_{L^{q}\left(\mathbb{R}^{N} \backslash B\right)}^{q}=\left|\mathbb{S}^{N-1}\right| \int_{r_{0}}^{\infty} \frac{r^{N-1} \lambda^{q}}{\left[1+\left(\lambda^{\frac{2}{N-s}} r\right)^{2}\right]^{N}} d r=\left|\mathbb{S}^{N-1}\right| \int_{\lambda^{N-s}}^{\infty} \frac{r^{N-1}}{\left(1+r^{2}\right)^{N}} d r
$$


This implies that $\lambda^{\frac{2}{N-s}} r_{0} \geq 1$ and therefore

$$
\begin{aligned}
\left\|U_{\lambda, 0}\right\|_{L^{q}\left(\mathbb{R}^{N} \backslash B\right)}^{q} & =\left|\mathbb{S}^{N-1}\right| \int_{\lambda^{N-s}}^{\infty} r_{0} \frac{r^{N-1}}{\left(1+r^{2}\right)^{N}} d r \\
& \geq 2^{-N}\left|\mathbb{S}^{N-1}\right| \int_{\lambda^{N-s}}^{\infty} r_{0} \frac{d r}{r^{N+1}}=\frac{\left|\mathbb{S}^{N-1}\right|}{N\left(2 r_{0}\right)^{N}} \lambda^{-q} .
\end{aligned}
$$

Combining (42) and (43), we conclude that

$$
d(u, \mathcal{M}) \geq \frac{C_{1}}{\lambda} \quad \text { with } C_{1}:=\sqrt{\mathcal{S}}(1-\rho)\left(\frac{\left|\mathbb{S}^{N-1}\right|}{N\left(2 r_{0}\right)^{N}}\right)^{\frac{1}{q}} .
$$

Using (35), (38) and (44), we find that

$$
\begin{aligned}
|u|_{w, \Omega} & \leq\left|c U_{\lambda, y}\right|_{w, \Omega}+\left|u-c U_{\lambda, y}\right|_{w, \Omega} \leq(1+\rho)\left|U_{\lambda, y}\right|_{w, \mathbb{R}^{N}}+\frac{1}{\sqrt{\mathcal{S}}}\left\|u-c U_{\lambda, y}\right\|_{s / 2} \\
& =\frac{1+\rho}{\lambda}|U|_{w, \mathbb{R}^{N}}+\frac{1}{\sqrt{\mathcal{S}}} d(u, \mathcal{M}) \leq C_{2} d(u, \mathcal{M})
\end{aligned}
$$

with $C_{2}:=\frac{(1+\rho)}{C_{1}}|U|_{w, \mathbb{R}^{N}}+\frac{1}{\sqrt{\mathcal{S}}}$. Combining this with (40), we thus obtain the claim with $C_{0}:=\max \left\{C_{2}, \frac{1}{\rho \sqrt{\mathcal{S}}}\right\}$.

Finally, Theorem 2 now simply follows by combining Theorem 1 and Proposition 3 and setting $C:=C_{0}^{-2}$.

Acknowledgement. U.S. National Science Foundation grant PHY-1068285 (R.F.) and German Science Foundation (DFG) grant WE 2821/4-1 (T.W.) is acknowledged. Shibing Chen wants to thank Robert McCann for helpful discussions.

\section{REFERENCES}

[1] Th. Aubin, Problèmes isopérimétriques et espaces de Sobolev, J. Differ. Geometry 11 (1976), 573- 598.

[2] T. Bartsch, M. Schneider and T. Weth, Multiple solutions to a critical polyharmonic equation, J. Reine Angew. Math. 571 (2004), 131-143.

[3] T. Bartsch, T. Weth and M. Willem, A Sobolev inequality with remainder term and critical equations on domains with topology for the polyharmonic operator, Calc. Var. Partial Differential Equations, 18 (2003), 253-268.

[4] W. Beckner, Sharp Sobolev inequalities on the sphere and the MoserTrudinger inequality, Ann. Math. 138 (1993), 213-242.

[5] G.Bianchi and H.Egnell, A note on the Sobolev inequality, J. Funct. Anal. 100 (1991), 18-24.

[6] H.Brezis and E.Lieb, Sobolev inequalities with remainder terms, J. Funct. Anal. 62 (1985), 73-86.

[7] E. A. Carlen, A. Figalli, Stability for a GNS inequality and the Log-HLS inequality, with application to the critical mass Keller-Segel equation, Preprint (2011), arXiv:1107.5976

[8] A. Cianchi, N. Fusco, F. Maggi, A. Pratelli, The sharp Sobolev inequality in quantitative form. J. Eur. Math. Soc. (JEMS) 11 (2009), no. 5, 1105-1139.

[9] W. Ding, On a conformally invariant elliptic equation. Commun. Math. Phys. 107 (1986), 331-335.

[10] J. Dolbeault, Sobolev and Hardy-Littlewood-Sobolev inequalities: duality and fast diffusion, Math. Res. Lett. 18 (2011), no. 6, 1037-1050

[11] J. Dolbeault, G. Toscani, Improved Sobolev's inequalities, relative entropy and fast diffusion equations, Preprint (2011), arXiv:1110.5175

[12] A. Figalli, F. Maggi, A. Pratelli, A mass transportation approach to quantitative isoperimetric inequalities. Invent. Math. 182 (2010), no. 1, 167-211. 
[13] N. Fusco, F. Maggi, A. Pratelli, The sharp quantitative isoperimetric inequality. Ann. of Math. (2) 168 (2008), no. 3, 941-980.

[14] F. Gazzola, H.C. Grunau, Critical dimensions and higher order Sobolev inequalities with remainder terms, Nonlin. Diff. Eq. Appl. 8 (2001), 35-44.

[15] P. Gérard, Description du défaut de compacité de l'injection de Sobolev. ESAIM : Control, Optimisation and Calculus of Variations 3 (1998), 213-233.

[16] R.A. Hunt, On $L(p, q)$ spaces, Enseignement Math. (2) 12 (1966), 249-276.

[17] T. Jin, J. Xiong, A fractional Yamabe flow and some applications. Preprint (2011), arXiv:1110.5664

[18] E. H. Lieb, Sharp constants in the Hardy-Littlewood-Sobolev and related inequalities. Ann. of Math.(2) 118 (1983), 349-374.

[19] E. H. Lieb, M. Loss, Analysis. Second edition. Graduate Studies in Mathematics 14, Amer. Math. Soc., Providence, RI, 2001.

[20] P.L.Lions, The concentration-compactness principle in the calculus of variations. The limit case. I, Riv. Mat. Iberoamericana 1 (1985), 145-201.

[21] P.L.Lions, The concentration-compactness principle in the calculus of variations. The limit case. II, Riv. Mat. Iberoamericana 1 (1985), 45-121.

[22] G.Lu and J.Wei, On a Sobolev inequality with remainder terms, Proc. Amer. Math. Soc. 128 (1999), 75-84.

[23] C. Morpurgo, Sharp inequalities for functional integrals and traces of conformally invariant operators, Duke Math. J. 114 (2002), 477-553.

[24] G. Rosen, Minimum value for $c$ in the Sobolev inequality, SIAM J. Appl. Math. 21 (1971), 30-32.

[25] G. Talenti, Best constant in Sobolev inequality, Ann. Mat. Pura Appl. 110 (1976), 353-372.

Department of Mathematics, University of Toronto, 40 St. George Street, Toronto, Ontario, Canada M5S 2E4

E-mail address: shibing.chen@utoronto.ca

Department of Mathematics, Fine Hall, Princeton University, Princeton, NJ 08544 (USA)

E-mail address: rlfrank@math.princeton.edu

Institut für Mathematik, Goethe-Universität, Robert-Mayer-Str. 10, 60054 FrankFURT (GERMANY)

E-mail address: weth@math.uni-frankfurt.de 